\title{
Sterilization Protocol in Orthodontic Practice: A Review
}

\author{
Sheetal Jankare ${ }^{1 *}$, Samsha S Surani ${ }^{2}$, Prafull Parchake ${ }^{1}$, Ekta Borkar ${ }^{3}$ and Abhishek Rathod ${ }^{4}$ \\ ${ }^{1}$ Assistant Professor, Government Dental College and Hospital, Mumbai, India \\ ${ }^{2}$ MDS Orthodontics and Dentofacial Orthopedics, Mumbai, India \\ ${ }^{3}$ MDS Oral Pathology and Microbiology, Mumbai, India \\ ${ }^{4}$ PG Student Orthodontics and Dentofacial Orthopedics, Mumbai, India \\ *Corresponding Author: Sheetal Jankare, Assistant Professor, Government Dental College and Hospital, Mumbai, India.
}

Received: October 10, 2019; Published: November 08, 2019

DOI: $10.31080 /$ ASDS.2019.03.0695

\begin{abstract}
Sterilization in Orthodontics and particularly in the entire dental practice is an important topic which requires special attention because both the patients and the practitioners have a substantial risk of spreading infections like hepatitis B, pneumonia, tuberculosis and HIV. Control of infection that spreads through various instruments and armamentarium used in the field of orthodontics and also in the dental practice is of utmost importance to prevent cross-infection. The present article reviews various recent methods of sterilization for an effective and efficient infection-free orthodontic practice.

Keywords: Sterilization; Orthodontics; Infection Control; Disinfection
\end{abstract}

\section{Introduction}

The field of dentistry deals with the diverse nature of oral flora which is rich in various aerobic and anaerobic bacteria. Individuals undergoing treatment in the dental office may be asymptomatic Hepatitis-B and Herpes carriers secreting viruses in saliva thereby transmitting diseases [1]. According to a survey by Feldman, Orthodontists have the second-highest incidence of contracting Hepatitis B among the dental practitioners [2]. Starnbach (1988) also suggests that the most common mode of transmission in dental offices is through the puncture wounds, skin abrasions, or lesions. Dental aerosols, splattering, and instrument contamination can also transmit the viruses, which can survive for several weeks at the room temperature [3].

The first general infection control for dentistry was published by the Centre for Disease Control and Prevention (CDC) in 1986 and is being updated every year. The main principle is to consider each patient as being infected because many infectious diseases can be present in an individual without signs and symptoms [4].
As an Orthodontist, our goals should be to reduce the number of pathogenic micro-organisms to the level which our body resistance may prevent disease and prevent cross-infection. Although many authors have previously published articles raising their concern about sterilization in orthodontic offices [1,4-6]. The present article reviews the various recent sterilization protocols about orthodontic instrumentation and armamentarium and along with the general infection control measures.

Primary goals of infection control in dental clinic are [1].

- To lower the risk of contamination by reducing the level of pathogens.

- To correct any break in aseptic technique.

- To use universal precautions with every patient (treat every patient and instrument as potentially infective).

- To protect patients and personnel from occupational infection. 
Before we proceed with the sterilization and disinfection for orthodontic practice it is essential to become acquainted with few of the commonly used terminologies [5].

1. Sterilization is a process by which an article, medium or surface is freed from all the pathogenic micro-organisms either in vegetative or spore form

2. Disinfection- Disinfection is the process of destroying or inhibiting most pathogenic micro-organisms and inactivating some viruses, hence reducing microbial contamination to the safety level.

3. Antisepsis- Application of chemicals on living tissue to avoid infection.

4. Asepsis- It means an environment free of germs which involves the destruction of all disease forming micro-organisms in the working environment.

5. Decontamination- Work against all kinds of germs to reduce microbial source in a number for protection from, unexpected contamination and infection is called decontamination.

\section{Areas of infection control}

Patient's history: During the initial appointment, a detailed and complete medical history to be taken from the patient, and in subsequent visits, updated accordingly.

Orthodontist and staff: It is crucial that the orthodontist and the dental staff must be vaccinated against tuberculosis, rubella,

Different Techniques of sterilization includes diphtheria, tetanus and most importantly, against HBV. Generally, orthodontist does not perform oral surgery, however, they should wear a lab coat, face mask and hand gloves as ageneral precautionary measure. Disposable gowns, surgical masks, protective eyeglasses and plastic face masks should be worn during procedures that are likely to splash blood, saliva and oral fluids [3].

Operating area: Chair, table, light handles, spittoon, three-way syringes etc., should be wiped frequently with $70 \%$ isopropyl alcohol after every patient [1].

\section{Instruments}

Instruments used in orthodontics can be of three categories according to Spaulding system: [6]

a) Critical: - Instruments that penetrate the mucosa should be sterilized. E.g. Molar bands, band remover plier, band pinching pliers, mini-implant placement kit etc.

b) Semi Critical: - Instruments that touch the mucosa should be sterilized whenever possible or treated with high-level disinfectants. E.g. Most of the orthodontic instruments, intra-oral mirrors, cheek retractors, and dental hand pieces, etc.

c) Least Critical: - Instruments that do not come in contact with mucous membrane such as bracket positioners, arch-forming pliers, torquing keys, bracket positioning gauges, bird beak plier etc. should be disinfected.

\begin{tabular}{|c|c|c|}
\hline Method of sterilization & Advantage & Disadvantage \\
\hline Hot Air Oven & $\begin{array}{l}\text { No corrosion. } \\
\text { Large capacity per cost. } \\
\text { Items are dry after the cycle. }\end{array}$ & $\begin{array}{l}\text { 1. } \quad \text { Longer sterilization time. } \\
\text { 2. } \quad \text { Cannot sterilize liquids. } \\
\text { 3. } \quad \text { May damage plastic and rubber items. }\end{array}$ \\
\hline Rapid Heat Sterilizer & $\begin{array}{l}\text { No corrosion. } \\
\text { Short cycle. } \\
\text { Items are dry after the cycle. }\end{array}$ & $\begin{array}{l}\text { Cannot sterilize liquids. } \\
\text { May damage plastic and rubber items. } \\
\text { Small capacity per cost. }\end{array}$ \\
\hline Autoclave & $\begin{array}{ll}\text { 1. } & \text { Good penetration. } \\
2 . & \text { Time-efficient. } \\
\text { 3. } & \text { Sterilize water-based liquids. } \\
\end{array}$ & $\begin{array}{l}\text { 1. } \quad \text { Non-Stainless items may corrode. } \\
\text { 2. } \quad \text { Closed containers cannot be used. } \\
\text { 3. } \quad \text { May damage plastic or rubber items. }\end{array}$ \\
\hline $\begin{array}{l}\text { Unsaturated Chemical Va- } \\
\text { pour Sterilization }\end{array}$ & Suitable method for orthodontic instruments & $\begin{array}{l}\text { Drawback: The odour, even though not toxic requires } \\
\text { adequate ventilation. }\end{array}$ \\
\hline Ethylene Oxide Sterilization & Suited for large institutions. & $\begin{array}{l}\text { 1. Slow procedure-- } 4 \text { hours at } 54 \mathrm{oC} \text { and } 12 \\
\text { hours at room temperature. } \\
\text { 2. Costly. } \\
\text { 3. If moisture is present it reacts with ethylene } \\
\text { oxide to form ethylene glycol coating. } \\
\text { 4. After sterilization articles should be aerated } \\
\text { for } 24 \text { hours for dissipation of gas. }\end{array}$ \\
\hline
\end{tabular}

Table 1 
Practical guideline for sterilization of the orthodontic instruments

Advice sheet issued for infection control in dentistry enlists three stages for sterilization as pre- sterilization cleaning, sterilization and storage [7].

1. Pre-Sterilization Cleaning: This process involves debridement of all instruments contaminated with blood, saliva and other impurities prior to undergoing a sterilization process. It is generally done by washing the instruments using detergents and brushes. However, in recent times ultrasonic cleanser and instrument washer have also been employed. Depending on the instrument loaded this procedure lasts between 10 - 20 minutes. At the end of pre-sterilization procedure, it is very important to eliminate any residual moisture on the instruments. Special consideration must be given to the joints and tips (serrated) of the instruments as it increases corrosion tendency in these instruments, especially while using certain methods of sterilization [8].

2. Sterilization- The method of sterilization depends not only on the anti-microbial effectiveness but also on the factors involving turnaround times which determine the requirement of prior pre sterilization, additional inventory and the effects of these protocols on instruments.

3. Storage- Sterilized instrument should be stored in dry, covered condition. Sterilized instruments should not be stored in a disinfectant or antiseptic solution. The instruments necessary for the treatment should be selected prior to treatment session. Care must be taken to avoid cross contamination if any additional instruments are needed.

Sterilization and disinfection of orthodontic instruments and materials

Orthodontist generally do not carry out extensive surgical procedures, however, patients can carry germs that may infect other patients. The use of proper sterilization techniques is important in day to day clinical practice because of professional, ethical and legal aspects [4].

\section{Disinfection of orthodontic brackets}

Reuse of the orthodontic brackets is not advisable because it affects the clinical performance of the brackets and also increases the chances of infection. Speera., et al. did a study to evaluate the effect of $0.01 \%$ chlorhexidine disinfectant solution on bond strength of metal and ceramic brackets and concluded that chlorhexidine does not have a significant effect on the metal and ceramic brackets adhesion ability. Thus, chlorhexidine is the recommended disinfectant for metal and ceramic brackets [9].

\section{Disinfection of orthodontic bands}

Stainless steel bands are most commonly used on molars during fixed orthodontic treatment.

Choosing an appropriate size requires several trials. During trying of bands if it is of not right size it should be decontaminated from saliva and blood, and autoclaved for future use [10].

Glass bead sterilizer has been shown to produce equally effective sterilization as that of autoclave [11-12]. The recommended protocol for sterilization of single molar band is $220^{\circ} \mathrm{C}$ for 5 seconds. If more than one band is placed at the same time the amount of time required is doubled. Other techniques for disinfecting bands, such as a 5 -second tap water rinse, 10-second soap scrub, 30-minute immersion in alcohol, and alcohol flame, were ineffective in killing bacteria or spores. However, alcohol flames were effective in preventing growth on orthodontic molar bands inoculated with staphylococcus albus [13].

Fulford (2003) described a method in which initial decontamination was done with enzymatic cleaning agent followed by steam sterilization or vacuum cycle bench top sterilizer. Enzymatic cleaning agent containing protease, amylase and lipase was used in order to breakdown and removal of residual proteins, fats and carbohydrates present in the saliva and blood [14].

General procedure for disinfection of orthodontic bands include

1. Ultrasonic scaling for 5-12 min.

2. Rinsing it with distilled water

3. Remove the excess moisture

4. Sterilize using dry heat or autoclave (as recommended)

5. Chemical immersion protocols should be limited to bands without prewelded attachments

6. Glass bead sterilization is only an option.

Disinfection of orthodontic pliers

The process of cleaning and sterilizing orthodontic instruments present special problems as these instruments have large hinge 
areas that are difficult to clean and sterilize. They also have cutting edges, sharp angles, and pointed ends that are easily damaged [15].

Also sterilization protocols employed for orthodontic pliers have been reported to affect the physical and mechanical properties and possess high risk of corrosion Autoclaving negatively affects orthodontic instruments causing blunting and corrosion of their sharp cutting edges. One of its major disadvantages is that it is time consuming. Hence, soaking in $1 \%$ sodium nitrate can be recommended as an alternative [16]. Pliers which are manufactured from stainless steel can be effectively sterilized using steam sterilization as it does not produce any deleterious effects. Jones found no significant difference in the appearance, corrosion, or efficiency of orthodontic pliers when comparing autoclaving with cold disinfection [17-18]. Chrome plated pliers appear more resistant to damage as compared to stainless steel [19]. Other methods such as microwave irradiation for $10 \mathrm{sec}$, glass bead sterilization for 30 seconds are recommended [20-21].

Prion protection [22].

Prions are extremely stable group of infectious agents that are resistance to conventional sterilization protocol. They are basically composed of proteins and hypothesized to infect and propagate by refolding abnormally into a structure which lead to conversion into abnormally structured protein. This conversion leads to extremely stable structure making it resistant to destruction by chemical and physical methods. The recommended protocol for prion include autoclave cycles at 1210 for 60 minutes or 1340 for at least 18 minutes or combination cycles involving hot air sterilization followed by autoclaving to improve the margin of the safety.

\section{Corrosion resistance}

Prior to dry-heat sterilization, if water drops or excess disinfectant is left on the pliers they can be severely damaged. Corrosion of these instruments is one of the few sterilization consequences that orthodontists face. Corrosion is an electrochemical event that metals undergo when reacting with an oxidant as a result of oxidation and reduction reactions. To prevent corrosion, orthodontic pliers should be dried with pressured air prior to sterilization. If they are not dried well, ion-reaction will create a loose layer of rust. Corrosion can also be prevented by oiling the joint surfaces with appropriate solutions. Studies suggest less corrosion with heat sterilization as compared to cold sterilization. These corroded pliers can be polished to restore its usability [23]. Wohlen Haus A at el in their study found ultrasound bath and thermal disinfection as the most effective method and therefore, they recommended it for the disinfection of orthodontic pliers [24].

\section{Disinfection of elastomeric chains}

Polyurethane elastomers are frequently used in orthodontics as ligature and chain. The unused parts of elastomeric ligatures are generally sterilized via cold sterilization since they are not heatresistant. Disinfection of these materials in a $5 \%$ glutaraldehyde solution for a period of 10 minutes is recommended. Various studies showed that repeated disinfection of the same elastic can accelerate the destruction of the cross links available in the long chain molecules of polyurethane polyesters. Sterilization of elastomeric ligatures inside the autoclave at $124 \mathrm{C}$ does not lead to permanent deformations or to increased shrinkage whereas in the case of dry-heat, their manipulation becomes more difficult [25]. Based on two different disinfectants, tensile strength and glass transformation temperature of elastomeric ligatures that are not disinfected are found significantly different than those that are exposed to phenol and glutaraldehyde [26]. A parallel observation was detected between the decrease in tensile strength as a result of exposure to disinfectants in study and the decrease in tensile strength in Jeffries and Fraunhofer's study [27].

Breakage of intermolecular links and glass transformation temperatures are decreased as a result of prolonged contact with disinfectants. Polyurethanes are not inert materials, and when they are exposed to enzymes, water, moisture and heat, they will absorb water and get destroyed. As a result of the plasticizer effect of disinfection solutions on polymer ligatures, decrease in tensile force and glass transformation temperature will occur [25]. It was proved in a study that disinfection by $0.12 \%$ chlorhexidine and $0.2 \%$ peracetic acid solution had no delirious effect on the properties of elastomeric chains [28].

\section{Sterilization of orthodontic markers}

Maryam at el did a comparative study using three different sterilization methods on orthodontic markers concluded that autoclaving and glutaraldehyde solution are the best methods for disinfecting orthodontic markers [29].

\section{Sterilization of orthodontic archwires}

Alavi., et al. studied effect of steam and dry heat sterilization on bending properties of Ni-Ti arch wires and found significant 
changes in the bending properties of super elastic $\mathrm{Ni}$-Ti arch wires. These wires showed decrease in super elasticity and decrease in applied force in both loading and unloading forces [30].

In contrast, various other researchers have demonstrated minimal or no effect on mechanical properties of stainless steel, $\mathrm{Ni}$ Ti and $\beta$ titanium arch wires following dry heat or cold sterilization method [31-39]. Also no significance difference was observed in release of the nickel ion concentration into saliva from recycled $\mathrm{Ni}$ TI wires after sterilization with dry heat or steam sterilization [40]. Stagger found no difference in mean tensile strength of stainless steel and sent alloy wire after five cycles of sterilization with autoclave or ethylene oxide sterilization [41].

Sterilization of orthodontic mini-implant

A single cycle of Autoclave sterilization has been recommended for mini-implant. Since, it has been shown repeated sterilization has delirious effect on its primary stability [42]. Gross suggested no significant biological changes even after four cycles insertion, removal and sterilization [43].

\section{Disinfection of removable appliances}

Disinfection of baseplates of removable orthodontic appliances by using $0.12 \%$ chlorhexidine spray once or twice a week reduces the contamination by Streptococcus Mutans on the acrylic surface [44-46].

Oral Safe is a germicide-deodorant that is harmless found to destroy $99 \%$ of microbes on removable appliances during 10 minutes of submersion. Now a three-minute procedure has been developed that combines the use of a germicide-deodorant with ultrasonic energy to kill 10 times more microorganisms than passive submersion, and to clean and thoroughly deodorize removable appliances [47].

In Lessa., et al's study, chlorhexidine gluconate, acetylpyridine chlorodyne and sterile water were compared in terms of their eliminating action on Streptococcus mutants. Antimicrobial solutions in spray form were used, and they were examined for causing any changes in the composition of acrylic or not. The results of this study suggested that both of the previously mentioned antimicrobial agents reduced contamination compared to sterile water, but chlorhexidine gluconate was found to be significantly more effective than acetylpyridine chlorodyne [48]. Fernanda compared peri guard, capicola and sterile water and found these antimicrobial agents are highly effective in reducing MS colonies/ biofilms on acrylic surfaces [49].
Disinfection of alginate impression and dental cast

All the impressions and bite records which are placed in patient's mouth should be properly rinsed to remove saliva, blood and debris and then disinfected by immersion in disinfecting product prior to handling over to the laboratory. Laboratories should have proper infection control.

\section{Impressions / casts}

Remove excess water

Dip the impression in a $0.525 \%$ sodium hypochlorite for 10 minutes and then place in a moisture proof sealed plastic bag. Dental casts from impressions may contain infectious microorganisms. Savage at el found that $2 \%$ glutaraldehyde was the most effective disinfectant with the least adverse effects on the physical properties on the cast. Although povidone-iodine caused a decrease in the compressive strength of the cast, it can be a sound alternative [50].

Commonly used disinfecting solutions used for alginate impressions are $2 \%$ glutaraldehyde and $1 \%$ sodium hypochlorite. The manufacturer prescription recommends immersing the alginate impression for not more than 10 minutes as it may cause alteration in the surface characteristics of the material. Newer alginate impression materials are commercially available as selfdisinfecting alginates [51-52].

1. Rinse the impression under running water on removal from the oral cavity.

2. Immerse them in disinfecting solution for 7-8 minutes (not more than 10 minutes).

3. Clean under running water to remove the disinfecting solution.

4. Pour the model.

Surface disinfection

Surfaces which cannot be sterilized must be disinfected effectively. These surfaces include air water spray, suction tips, head rests, drawers, head rest and arms. These surfaces can be disinfected using 1\% sodium hypochlorite or solutions containing $70 \%$ alcohol. Iodine solutions can be used for disinfection which are cheap, easy stored and highly effective. The only disadvantage is the staining of iodine [4].

\section{Conclusion}

"Prevention is better than cure" a proverb well suited to sterilization. Considering the enormity of challenge that infectious 
agent pose against us also their nature to continuously evolve with time, implementation and execution of effective infection control protocols amongst health care professionals including us is essential. Thorough understanding of the application of sterilization will help ensure safety from the invisible but deadly world of microbial pathogens Hence utilization of proper sterilization, disinfectants and aseptic procedures help us achieve the safety of our professional demands.

\section{Bibliography}

1. Shilpa kalra., et al. "Infection control in orthodontics". Journal of Orthodontics and Endodontics 1 (2015): 1-12.

2. Ronald E Feldman and Eugene RS "Hepatitis in dental professionals". Journal of the American Medical Association 232.12 (1975): 1228-1232.

3. Starnbach $\mathrm{H}$ and Biddle P. "A pragmatic approach to asepsis in the orthodontic office". The Angle Orthodontist 0 (1988): 6366.

4. Alev Aksoy., et al. "Sterilization and disinfection in orthodontics". Intechopen Journal (2011).

5. Ananthnarayan and paniker's Textbook of microbiology 10th edition

6. Spaulding EH. "Chemical disinfection of medical and surgical materials. In: Lawrence C, Block SS, editors. Disinfection, sterilization, and preservation". Philadelphia: Lea and Febiger (1968): 517-531.

7. Advice sheet, infection control in dentistry, department of health, United Kingdom (2005).

8. Vinay P., et al. "Sterilization methods in orthodontics-A review". International Journal of Dental Clinics 3.1 (2011): 4447.

9. Speera C., et al. "Bond Strength of Disinfected Metal and Ceramic Brackets". The Angle Orthodontist 75.5 (2005): 836-842.

10. Benson and Douglas. "Sterilization and Disinfection in Orthodontics". Intech Open (2007).

11. Saugat R., et al. "Reliability of Glass Bead Sterilization for Tried-in Orthodontic Bands". The Journal of Indian Orthodontic Society 45.4 (2011): 189-192.

12. Benso PE and Douglas CWI. "Decontamination of Orthodontic Bands following Size determination and Cleaning". Journal of Orthodontics 34 (2007):18-24.
13. Smith GE. "Glass bead sterilization of orthodontic bands". American Journal of Orthodontics and Dentofacial Orthopedics 90.3 (1986): 243-249.

14. Fulford MR., et al. "Decontamination of tried-in orthodontic molar bands". European Journal of Orthodontics 25.6 (2003): 621-622.

15. Arif Yezdani., et al. "Orthodontic instrument sterilization with microwave irradiation". Journal of Pharmacy and Bioallied Sciences 7.1 (2015).

16. Jeevan M Khatri., et al. "Sterilization and orthodontics: A literature review". International Journal of Orthodontic Rehabilitation 8.4 (2017): 141-146.

17. Malcolm Jones and Kevin Pizzaro. "The effect of routine steam autoclaving on orthodontic pliers". European Journal of Orthodontics 15 (1993): 281-290.

18. Vendrel RJ and Hayden CL. "Effect of steam versus dry heat sterilization on the wear of orthodontic ligature cutting pliers". American Journal of Orthodontics and Dentofacial Orthopedics 121 (2002): 467-471.

19. Jones ML. "An initial assessment of the effect on orthodontic pliers of various sterilization/disinfection regimes". British Journal of Orthodontics 16 (1989): 251-258.

20. Miller JA and Harrower KM. "A novel method of sterilizing orthodontic instruments". Australasian Orthodontic Journal 12.3 (1992):151-152.

21. Yezdani A and Mahalakshmi K. "Orthodontic instrument sterilization with microwave irradiation". Journal of Pharmacy and Bioallied Sciences 7.1 (2015): S111-S115.

22. Arshad., et al. "Instrument sterilization in orthodontic clinic: A review". Journal of Contemporary Medicine and Dentistry 3.3 (2015).

23. Wichelhaus A and Brauchle G. "Corrosion of orthodontic pliers using different sterilization procedures". Journal of Orofacial Orthopedics 65.6 (2004): 501-511.

24. Wichelhaus A., et al. "Effective Disinfection of Orthodontic Pliers". Journal of Orofacial Orthopedics 67 (2006): 316-336.

25. Mayberry., et al. "Effects of Disinfecting Procedures on Elastomeric Ligatures". Journal of Clinical Orthodontics 3 (1996): 49-51. 
26. Evangelista., et al. "Effect of Disinfecting Solutions on the Mechanical Properties of Orthodontic Elastomeric Ligatures Maylani B. Evangelistaa; David W. Berzinsb; Peter Monaghanc Angle Orthodontist 77.4 (2007).

27. Jeffries and Fraunhofer's study Jeffries CL and von Fraunhofer JA. "The Effects of \%2 Alkaline Glutaraldehyde Solution on the Elastic Properties of Elastomeric Chain". The Angle Orthodontist 61 (1991): 25-33.

28. Karina A., et al. "Force decay in orthodontic elastomeric chains after immersion in disinfection solutions". Brazilian Journal of Oral Sciences 4.13 (2014): 266-269.

29. Omidkhoda Maryamkus., et al. "Comparison of three different sterilization and disinfection methods on orthodontic markers". Journal of Orthodontic Science 5.1 (2016): 14-17.

30. Shiva Alavi. "Effects of steam and dry heat sterilization on bending properties of Ni-Ti wires". Orthodontic Waves 68 (2009): 123-128.

31. Smith GA and von fraunhofer J A. "The effect of clinical use and sterilization on selected orthodontic archwires". American Journal of Orthodontics and Dentofacial Orthopedics 102.2 (1992):153-159.

32. Pernier C., et al. "Influence of autoclave sterilization on the surface parameters and mechanical properties of six orthodontic wires". European Journal of Orthodontics 27.1 (2005): 72-81.

33. M Oshagh. "Autoclaving and clinical recycling: Effects on mechanical properties of orthodontic wires". Indian Journal of Dental Science 23.5 (2012): 638-642.

34. Joji Isac. "Effects of clinical recycling on mechanical properties of three commonly used types of orthodontic archwires". Saudi Journal of Oral and Dental Research 1.3 (2016): 124-136.

35. Lee SH and Chang Y. "Effects of recycling on the mechanical properties and the surface topography of nickel titanium alloy wires". American Journal of Orthodontics and Dentofacial Orthopedics 120.6 (2001): 654-663.

36. Sunil Kapila. "Effects of clinical recycling on mechanical properties of nickel- titanium alloy wires". American Journal of Orthodontics and Dentofacial Orthopedics 100.5 (1991): 428435.
37. Sunil Kapila. "Load-deflection characteristics of nickel-titaniu $\mathrm{n}$ alloy wires after clinical recycling and dry heat sterilization". American Journal of Orthodontics and Dentofacial Orthopedics 102 (1992): 120-126.

38. Mayhew MJ and Kusy RP. "Effects of sterilization on the mechanical properties and the surface topography of nickel-titanium arch wires". American Journal of Orthodontics and Dentofacial Orthopedics 93.3 (1988): 232-236.

39. Buckthal J and Kusy RP. "Effects of cold disinfectants on the mechanical properties and the surface topography of nickeltitanium arch wires". American Journal of Orthodontics and Dentofacial Orthopedics 94.2 (1988): 117-122.

40. Poosti M., et al. "Are more nickel ions released from NiTi wires after sterilisation?" Australian Orthodontic Journal 25.1 (2009): 30-33.

41. Stagger J A and Margeson S. "The effects of sterilization on the tensile strength of orthodontic wires". The Angle Orthodontist 63.20 (1993): 141-144.

42. Akyalcin S., et al. "Effects of repeated sterilization cycles on primary stability of orthodontic mini-screws". The Angle Orthodontist 83.3 (2013): 674-679.

43. Gross JM., et al. "Mini-implants for orthodontic anchorage:surface analysis after redrilling and sterilizationAn vitro study". The Journal of Contemporary Dental Practice 17.4 (2016): 300-305.

44. Amitha $\mathrm{H}$ and Munshi AK. "Effect of chlorhexidine gluconate mouth wash on the plaque microflora in children using intra oral appliance". The Journal of Clinical Pediatric Dentistry 20.1 (1995): 23-29.

45. Petit H., et al. "Disinfection of Removable Appliances". Journal of Clinical Orthodontics 19.4 (1985): 293-295.

46. Peixoto IT., et al. "Evaluation of home disinfection protocols for acrylic baseplates of removable orthodontic appliances: A randomized clinical investigation". American Journal of Orthodontics and Dentofacial Orthopedics 140.1 (2011): 51-57.

47. Ivanovski S., et al. "Disinfection of dental stone casts: antimicrobial effects and physical property alterations". Dental Materials 11.1 (1995): 19-23. 
48. Lessa FCR., et al. "In-vivo Evaluation of the Bacterial Contamination and Disinfection of Acrylic Baseplates of Removable orthodontic Appliances". American Journal of Orthodontics and Dentofacial Orthopedics 131 (2007): 705.e11- 705.e17.

49. Fernanda CR and Carla Enoki. "In-vivo evaluation of the bacterial contamination and disinfection of acrylic baseplates of removable orthodontic appliances". American Journal of Orthodontics and Dentofacial Orthopedics 131.6 (2007): 705. e11-705.e17.

50. Blair F and Wassell R. "A survey of the methods of disinfection of dental impressions used in dental hospitals in the United Kingdom". British Dental Journal 180.10 (1996): 369-375.

51. Choudhury GK., et al. "Disinfectant Efficacy of 0.525\% Sodium Hypochlorite and Epimax on Alginate Impression Material". The Journal of Contemporary Dental Practice 19.1 (2018): 113116.

52. Ivanovski S., et al. "Disinfection of dental stone casts: antimicrobial effects and physical property alterations". Dental Materials 11.1 (1995): 19-23.

Volume 3 Issue 12 December 2019

(C) All rights are reserved by Sheetal Jankare., et al. 